\title{
Association between the serum estrone-to- estradiol ratio and parameters related to glucose metabolism and insulin resistance in women with polycystic ovary syndrome
}

\author{
Nayoung Kim', Sungwook Chun ${ }^{2}$ \\ 'Department of Obstetrics and Gynecology, Pusan National University Yangsan Hospital, Yangsan; 'Department of Obstetrics and Gynecology, Inje \\ University Haeundae Paik Hospital, Inje University College of Medicine, Busan, Korea
}

Objective: We aimed to evaluate associations between the ratio of serum estrone (E1) to estradiol (E2) and parameters related to serum glucose metabolism and insulin resistance in women with polycystic ovary syndrome (PCOS).

Methods: In total, 133 women between the ages of 18 and 35 diagnosed with PCOS were enrolled in this study. All participants with PCOS underwent blood tests to determine hormonal and biochemical metabolic parameters and a standard 2-hour 75-g oral glucose tolerance test. They were divided into two groups according to the serum E1-to-E2 ratio: group 1 (E1/E2 ratio <2.0) and group 2 (E1/E2 ratio $\geq 2.0$ ).

Results: In the comparative analysis, the waist-to-hip ratio (WHR) was the only clinical variable that was significantly different between the two groups. Patients with a higher E1/E2 ratio showed higher fasting insulin levels, homeostasis model for insulin resistance, and postprandial glucose level at 2 hours (PPG2). In a correlation analysis, only PPG2 was significantly related to the serum E1/E2 ratio. However, after controlling for the confounding effects of body mass index (BMI) and WHR, fasting glucose was also significantly correlated with the serum E1/E2 ratio.

Conclusion: Women with PCOS with a higher serum E1/E2 ratio were found to be more likely to show higher fasting insulin and postprandial glucose levels. Significant correlations were found between the serum E1/E2 ratio and both fasting and postprandial serum glucose levels after adjusting for BMI and WHR in women with PCOS.

Keywords: Estradiol; Estrone; Glucose; Insulin resistance; Polycystic ovary syndrome

\section{Introduction}

Polycystic ovary syndrome (PCOS) is regarded as the most common endocrine disorder in women of reproductive age [1,2]. The prevalence of PCOS in women of reproductive age is approximately $3 \%-10 \%$. PCOS is one of the major causes of infertility with anovulation, and it is present in approximately $25 \%-30 \%$ of patients with in-

Received: April 28, 2021 · Revised: August 2, 2021 · Accepted: August 27, 2021 Corresponding author: Sungwook Chun

Department of Obstetrics and Gynecology, Inje University Haeundae Paik Hospital, 875 Haeun-daero, Haeundae-gu, Busan 48108, Korea

Tel: +82-51-797-2020 Fax: +82-51-797-2030 E-mail:wooki1974@empal.com

This is an Open Access article distributed under the terms of the Creative Commons Attribu tion Non-Commercial License (http://creativecommons.org/licenses/by-nc/4.0/) which permits unrestricted non-commercial use, distribution, and reproduction in any medium, provided the original work is properly cited. fertility [1].

The common characteristic clinical features of PCOS are amenorrhea or oligomenorrhea, inappropriate hormonal secretion (including hyperandrogenism), and abnormal metabolic status [1-3]. Hormonal imbalances besides hyperandrogenism in PCOS include increased luteinizing hormone levels, a reversed ratio of luteinizing hormone to follicle-stimulating hormone, increased anti-Müllerian hormone levels, mild prolactin elevation, increased inhibin and estrone (E1) levels, and a reversal of the ratio of E1 to estradiol (E2) (E1/ E2 ratio) $[1,2]$. Common metabolic problems associated with PCOS are glucose intolerance, type 2 diabetes mellitus (T2DM), central obesity, insulin resistance, and hyperinsulinemia [1-6].

Insulin resistance and hyperinsulinemia are the cardinal factors involved in the pathogenesis of PCOS $[7,8]$, and the reported preva- 
lence of insulin resistance in women with PCOS varies depending on race, ethnicity, and nationality [9-14]. Obese women with PCOS are characterized by insulin resistance, which increases the risk of metabolic and cardiovascular diseases [15]. In obese patients with PCOS, insulin resistance is higher than in non-obese patients with PCOS [16], but even in non-obese patients, insulin resistance is higher than that of controls $[17,18]$. Insulin resistance is defined as a state of subnormal to abnormal glucose utilization and homeostasis under normal concentrations of insulin production, and PCOS with obesity commonly amplifies the degree of these metabolic abnormalities $[19,20]$. Several assays can be used to evaluate insulin resistance, including the hyperinsulinemic clamp, homeostasis model for insulin resistance (HOMA-IR), fasting glucose-to-insulin ratio (GIR), quantitative insulin sensitivity check index (QUICKI), and the results of oral glucose tolerance testing (OGTT) [2,21,22]. The Endocrine Society Clinical Practice Guidelines [21] recommend the use of OGTT to screen adolescents and adult women with PCOS, who are at high risk for impaired glucose tolerance and T2DM. The OGTT is a standard diagnostic tool for impaired glucose tolerance and T2DM [2,21].

Elevated serum E1 levels and the resulting reversal of the E1/E2 ratio are common abnormal hormonal characteristics in patients with PCOS $[1,2,6,23]$. In women with PCOS, aromatase and 17-ß hydroxysteroid dehydrogenase activities are increased in cumulated peripheral fat cells along with increased peripheral aromatization and weight gain. E2 levels remain in the follicular phase range without mid-cycle level changes; on the contrary, E1 levels increase because of peripheral aromatization in response to increased androstenedione levels [24-26]. In a chronic hyperestrogenic state with reversal of the E1/E2 ratio, hormonal status and outcomes are unopposed by progesterone $[1,23]$.

Several studies have explored the relationship between hormonal characteristics and insulin resistance-related parameters in women with PCOS [27-30]. A reversed E1/E2 ratio is a distinctive hormonal characteristic of PCOS; however, to our knowledge, no study has been conducted to evaluate the relationships between an increased E1/E2 ratio and parameters related to glucose and insulin metabolism in PCOS. The aim of the present study was to evaluate whether the serum E1/E2 ratio is related to other metabolic parameters associated with insulin resistance in women with PCOS.

\section{Methods}

\section{Subjects}

This study was approved by the Institutional Review Board of Inje University Haeundae Paik Hospital (IRB No. 129792-2014-035), and patient's informed consent in this study was waived by the IRB. All patients were newly diagnosed with PCOS at 18-35 years of age from January 2010 to December 2013 at the above-mentioned university hospital. The patients were diagnosed with PCOS on the basis of the 2003 Rotterdam criteria. All patients who met at least two of the three criteria, including (1) oligo-anovulation, (2) biochemical and/or clinical signs of hyperandrogenism (e.g., hirsutism, acne, androgenic alopecia), and (3) ultrasonographically identified polycystic ovarian morphology (PCOM) of at least 1 ovary, were diagnosed with PCOS after excluding other diseases or etiologies [31]. Using the transvaginal or transrectal ultrasound approach, PCOM was defined as an ovarian volume of over $10 \mathrm{~cm}^{3}$ and/or the presence of over 12 follicles (2-9 mm in size). Pelvic ultrasonography (through the vagina or rectum) for assessing PCOM was conducted in the early follicular phase using a Voluson LOGIQ S7 (GE Ultrasound Korea, Seongnam, Korea) equipped with a transvaginal probe with a frequency range of 3.6-9 MHz, and all ultrasound examinations were conducted by the same reproductive endocrinologist. Oligo-anovulation was estimated on the basis of menstrual history and the presence of amenorrhea or oligomenorrhea. Amenorrhea was defined as a menstrual cycle interval of over 90 days without menstruation, and oligomenorrhea was defined as an interval of over 35 days. The most common clinical sign of hyperandrogenism is the presence of hirsutism, which was identified using a modified Ferriman-Gallwey score $>6$, based on a previous study of hirsutism in Korean women [32]. Biochemical hyperandrogenism was confirmed by elevated serum androgen concentration beyond the $95 \%$ confidence limits in the control group of a previous study (total testosterone $>0.68 \mathrm{ng} / \mathrm{mL}$ and/or free testosterone $>1.72 \mathrm{pg} / \mathrm{mL}$ ) [9,32]. Patients with a previous history of diagnosed diabetes, thyroid disease, hyperprolactinemia, or ovarian surgery were excluded. Patients who were taking oral contraceptives with or without prescriptions within the last 6 months and anti-diabetic drugs, including insulin sensitizers, were also excluded. All patients were divided into two groups based on the serum E1/E2 ratio: group 1 (E1/E2 ratio <2.0) and group 2 (E1/E2 ratio $\geq 2.0$ ).

\section{Clinical and biochemical measurements}

All clinical variables of the study participants were assessed when they first visited the outpatient department. Blood samples for biochemical laboratory analyses were taken from all subjects in the early follicular phase after overnight fasting. Serum E1 was measured using the Dsl-8700 Estrone ELISA kit (Beckman Coulter, Brea, CA, USA) and serum E2 was measured using Elecsys Estradiol II (Roche, Indianapolis, IN, USA) [23]. Serum insulin and glucose levels were analyzed using an Elecsys Insulin assay (Roche) and an L-Type Glul device (Wako, Osaka, Japan), respectively. Cholesterol and triglyceride levels were measured using Pureauto S (Sekisui, Tokyo, Japan), and serum high-density lipoprotein and low-density lipoprotein levels were 
measured using Cholestest (Sekisui) [30]. Both intra- and inter-assay coefficients of variation for all assays were below $8 \%$.

\section{Assessment of insulin resistance}

After overnight fasting, serum glucose and insulin levels were checked. Glucose levels at 60 minutes and 120 minutes after glucose ingestion during a 2-hour 75-g OGTT were measured. The fasting glucose level and postprandial glucose level at 2 hours (PPG2) were analyzed using an L-Type Glul device (Wako, Osaka, Japan). The insulin resistance parameters included HOMA-IR, QUICKI, and GIR. The GIR was calculated by dividing the glucose value $(\mathrm{mg} / \mathrm{dL})$ by the insulin value $(\mu \mathrm{U} / \mathrm{mL})$; HOMA-IR was calculated as fasting glucose $(\mathrm{mg} / \mathrm{dL}) \times$ fasting insulin $(\mu \mathrm{U} / \mathrm{mL}) / 405$; and QUICKI was calculated as $1 /\{$ log[insulin value $(\mu \mathrm{U} / \mathrm{mL})]+\log [$ glucose value $(\mathrm{mg} / \mathrm{dL})]\}$.

\section{Statistical analysis}

All values are expressed as mean \pm standard deviation. All statistical analyses were performed using SPSS ver. 18.0 (SPSS Inc., Chicago,
IL, USA). The paired $t$-test was used to compare clinical and biochemical parameters, including hormonal and glucose and insulin metabolism-related parameters, between the two groups categorized by the E1/E2 ratio. The correlations between the serum E1/E2 ratio and insulin resistance-related parameters were analyzed with Pearson correlation coefficients, and partial correlation coefficients were used after adjusting for body mass index (BMI) and the waist-to-hip ratio (WHR). In all analyses, $p$-values $<0.05$ were considered to indicate statistical significance.

\section{Results}

The mean E1/E2 ratio in group $1(n=74)$ was $1.31 \pm 0.42$ and that in group $2(n=59)$ was $3.23 \pm 1.40$. The comparisons of anthropometric parameters and serum hormonal levels, including E1 and E2, of the two groups are shown in Table 1. Among various clinical parameters, the WHR was the only parameter that was significantly different between the two groups $(p=0.010)$. As shown in Table 2, the

Table 1. Comparison of clinical characteristics between the two groups defined according to the serum E1-to-E2 ratio in women with polycystic ovary syndrome

\begin{tabular}{lccc}
\hline Variable & Group 1 $(\mathrm{n}=74)$ & Group 2 $(\mathrm{n}=59)$ & $p$-value \\
\hline Age $(\mathrm{yr})$ & $27.35 \pm 5.69$ & $26.54 \pm 5.29$ & 0.402 \\
Parity & $0.23 \pm 0.54$ & $0.20 \pm 0.61$ & 0.792 \\
Height $(\mathrm{cm})$ & $162.35 \pm 5.18$ & $160.85 \pm 5.36$ & 0.103 \\
Body weight $(\mathrm{kg})$ & $55.67 \pm 14.10$ & $57.78 \pm 12.65$ & 0.371 \\
Body mass index $\left(\mathrm{kg} / \mathrm{m}^{2}\right)$ & $21.10 \pm 5.22$ & $22.31 \pm 4.60$ & 0.165 \\
Waist-to-hip ratio & $0.78 \pm 0.06$ & $0.81 \pm 0.06$ & 0.010 \\
E1/E2 ratio & $1.31 \pm 0.42$ & $3.23 \pm 1.40$ & $<0.001$ \\
\hline
\end{tabular}

Values are presented as mean \pm standard deviation. Group 1, E1/E2 <2.0; group 2, E1/E2 $\geq 2.0$. A $p$-value was obtained by paired sample $t$-test. E1, estrone; E2, estradiol.

Table 2. Comparison of insulin resistance-related parameters between the two groups defined according to the serum E1-to-E2 ratio in women with polycystic ovary syndrome

\begin{tabular}{lccc}
\hline Variable & Group1 $(\mathrm{n}=74)$ & Group2 $(\mathrm{n}=59)$ & $p$-value \\
\hline Fasting insulin $(\mu \mathrm{lU} / \mathrm{mL})$ & $6.87 \pm 5.85$ & $9.60 \pm 8.43$ & 0.047 \\
Fasting glucose $(\mathrm{mg} / \mathrm{dL})$ & $91.48 \pm 20.28$ & $94.05 \pm 19.36$ & 0.468 \\
PPG2 (mg/dL) & $103.97 \pm 29.07$ & $120.91 \pm 56.09$ & 0.034 \\
HOMA-IR (fasting) & $1.57 \pm 1.36$ & $2.46 \pm 2.93$ & 0.044 \\
GIR (fasting) & $18.81 \pm 9.98$ & $16.46 \pm 11.76$ & 0.243 \\
QUICKI (fasting) & $0.37 \pm 0.04$ & $0.36 \pm 0.05$ & 0.089 \\
Cholesterol $(\mathrm{mg} / \mathrm{dL})$ & $170.86 \pm 28.41$ & $178.22 \pm 31.92$ & 0.167 \\
Triglyceride $(\mathrm{mg} / \mathrm{dL})$ & $89.55 \pm 78.11$ & $105.20 \pm 97.98$ & 0.313 \\
HDL $(\mathrm{mg} / \mathrm{dL})$ & $58.46 \pm 12.30$ & $58.31 \pm 14.75$ & 0.947 \\
LDL $(\mathrm{mg} / \mathrm{dL})$ & $95.65 \pm 25.18$ & $98.19 \pm 31.09$ & 0.615 \\
\hline
\end{tabular}

Values are presented as mean \pm standard deviation. Group 1, E1/E2 <2.0; group 2, E1/E2 $\geq 2.0$. A $p$-value was obtained by paired sample $t$-test. E1, estrone; E2, estradiol; PPG2, postprandial glucose level at 2 hours; HOMA-IR, homeostasis model assessment of insulin resistance; GIR, glucose-to-insulin ratio; QUICKI, quantitative insulin sensitivity check index; HDL, high-density lipoprotein; LDL, low-density lipoprotein. 
fasting insulin level, HOMA-IR, and PPG2 were significantly higher in group 2 (E1/E2 ratio $\geq 2.0$ ) than in group 1 .

In the correlation analysis, only PPG2 was significantly correlated with the serum E1/E2 ratio (Table 3). However, after adjusting for BMI and WHR (as anthropometric parameters known to be closely related to insulin resistance), postprandial and fasting glucose levels were significantly correlated with the serum E1/E2 ratio.

\section{Discussion}

A reversed E1/E2 ratio is a distinctive hormonal characteristic of PCOS $[1,2]$. The authors recently reported that serum E1 levels and the E1/E2 ratio were correlated with blood androgen levels, and in particular, the E1/E2 ratio was significantly correlated with the serum free testosterone level $(r=0.260, p=0.003)$ [23]. Insulin resistance and hyperinsulinemia are the cardinal factors involved in the pathogenesis of PCOS, which is associated with a high risk of glucose intolerance and T2DM [1-6,9,19-21]. However, to our knowledge, studies evaluating the relationship between increased serum E1 levels and parameters related to glucose and insulin metabolism are still lacking. Moreover, no previous study has evaluated the correlation between an increased E1/E2 ratio and the parameters related to insulin resistance in PCOS. This may be due to the high cost of commercial kits for determining E1 and the need for more complex laboratory techniques other than hormonal assays [23]. To the best of our knowledge, this is the first study conducted to evaluate the association between the serum E1/E2 ratio and parameters related to insulin and glucose metabolism in women with PCOS, and the results of

Table 3. Correlations of insulin resistance-related parameters with the serum E1-to-E2 ratio in women with polycystic ovary syndrome

\begin{tabular}{lcccc}
\hline Variable & $r$ & $p$-value & $r^{\mathrm{a}}$ & $p$-value \\
\hline Fasting insulin $(\mu \mathrm{lU} / \mathrm{mL})$ & 0.154 & 0.095 & 0.143 & 0.162 \\
Fasting glucose $(\mathrm{mg} / \mathrm{dL})$ & 0.123 & 0.170 & 0.270 & 0.007 \\
PPG2 (mg/dL) & 0.251 & 0.005 & 0.308 & 0.002 \\
HOMA-IR (fasting) & 0.161 & 0.081 & 0.192 & 0.060 \\
GIR (fasting) & -0.101 & 0.278 & -0.037 & 0.192 \\
QUICKI (fasting) & -0.156 & 0.092 & -0.119 & 0.246 \\
Cholesterol (mg/dL) & 0.114 & 0.197 & 0.146 & 0.141 \\
Triglyceride (mg/dL) & 0.085 & 0.336 & 0.065 & 0.511 \\
HDL (mg/dL) & 0.040 & 0.649 & 0.085 & 0.389 \\
LDL (mg/dL) & 0.037 & 0.672 & 0.095 & 0.339 \\
\hline
\end{tabular}

$r$, Pearson correlation coefficient; $r^{a}$, partial correlation coefficient adjusted by body mass index and waist-to-hip ratio.

E1, estrone; E2, estradiol; PPG2, postprandial glucose level at 2 hours; HOMA-IR, homeostasis model assessment of insulin resistance; GIR, glucose-to-insulin ratio; $\mathrm{QUICKI}$, quantitative insulin sensitivity check index; $\mathrm{HDL}$, high-density lipoprotein; LDL, low-density lipoprotein. our study suggest that the serum E1/E2 ratio is significantly related to both fasting and postprandial serum glucose levels in women with PCOS, after adjusting for confounding anthropometric factors.

Both fasting and postprandial glucose levels are major factors involved in insulin resistance, but the site of insulin resistance is known to be different between patients with abnormal fasting glucose and those with abnormal postprandial glucose levels according to previous clinical studies [33-37]. Individuals with impaired fasting glucose mainly show hepatic insulin resistance with normal muscle insulin sensitivity; on the contrary, those with impaired glucose tolerance typically show muscle insulin resistance with normal hepatic insulin sensitivity [34]. In the present study, the serum E1/E2 ratio was significantly related to both fasting and postprandial serum glucose levels after adjusting for BMI and WHR, suggesting that the E1/E2 ratio is related to both muscle insulin resistance and hepatic insulin resistance. Increased levels of androgenic precursors in theca cells induce the increased production of androstenedione, which is converted by $17 \beta$-hydroxysteroid dehydrogenase to testosterone or E1 by aromatization $[6,24,26]$. Aromatase and $17 \beta$-hydroxysteroid dehydrogenase activities occur in fat cells and ovarian theca cells; thus, weight gain leads to increased peripheral aromatization of androstenedione [6,24]. In PCOS, increased production of androstenedione with increased peripheral aromatization triggers an increase in serum E1 levels, which is frequently accompanied by weight gain and an increase in the WHR. In the present study, the WHR was significantly different between the two groups categorized on the basis of the E1/E2 ratio, which is partially consistent with the previous studies $[6,24,26]$. However, there was no significant difference in BMI between the two groups, which was contrary to what we had hypothesized.

In the present study, the subjects were divided into two groups based on an E1/E2 ratio of 2.0, which approximately corresponds to the average E1/E2 ratio of the participants in this study (2.16 \pm 1.37 ).

The limitations of our study are its retrospective study design, a relatively small sample size from a single institution, and the inclusion of only a single ethnic group (Korean women) among the study participants. Additionally, the lack of postload insulin data could be a crucial drawback of the present study. Further studies with a much larger sample size and cohorts with various ethnicities and races are needed to clarify these preliminary findings, and comparative studies with healthy controls could confirm these preliminary results.

In conclusion, women with PCOS with higher serum E1/E2 ratios were more likely to show higher fasting insulin and postprandial glucose levels. The serum E1/E2 ratio was significantly related to fasting and postprandial serum glucose levels after adjusting for BMI and WHR in women with PCOS. On the basis of this result, the serum E1/ E2 ratio may be a feasible hormonal marker that reflects the status of 
insulin and glucose metabolism in women with PCOS; however, additional studies are needed to corroborate our results so that they can be applied clinically.

\section{Conflict of interest}

No potential conflict of interest relevant to this article was reported.

\section{ORCID}

$\begin{array}{ll}\text { Nayoung Kim } & \text { https://orcid.org/0000-0003-2294-1592 } \\ \text { Sungwook Chun } & \text { https://orcid.org/0000-0002-9948-0360 }\end{array}$

\section{Author contributions}

Conceptualization: all authors. Data curation: all authors. Formal analysis: all authors. Methodology: all authors. Project administration: SC. Visualization: SC. Writing-original draft: all authors. Writingreview \& editing: all authors.

\section{References}

1. Kuzbari O, Doralis J, Peterson CM. Endocrine disorders. In: Berek JS. editor. Berek \& Novak's gynecology. 15th ed. Philadelphia: Lippincott Williams \& Wilkins; 2012. p. 1075-80.

2. Fritz MA, Speroff L. Clinical gynecologic endocrinology and infertility. 8th ed. Philadelphia: Lippincott Williams \& Wilkins; 2011. p. 501-18.

3. Broekmans FJ, Knauff EA, Valkenburg O, Laven JS, Eijkemans MJ, Fauser BC. PCOS according to the Rotterdam consensus criteria: Change in prevalence among WHO-II anovulation and association with metabolic factors. BJOG 2006;113:1210-7.

4. Diamanti-Kandarakis $E$, Dunaif A. Insulin resistance and the polycystic ovary syndrome revisited: an update on mechanisms and implications. Endocr Rev 2012;33:981-1030.

5. Yildizhan B, Anik Ilhan G, Pekin T. The impact of insulin resistance on clinical, hormonal and metabolic parameters in lean women with polycystic ovary syndrome. J Obstet Gynaecol 2016;36:8936.

6. Ehrmann DA. Polycystic ovary syndrome. N Engl J Med 2005;352: 1223-36.

7. Ehrmann DA, Liljenquist DR, Kasza K, Azziz R, Legro RS, Ghazzi $\mathrm{MN}$, et al. Prevalence and predictors of the metabolic syndrome in women with polycystic ovary syndrome. J Clin Endocrinol Metab 2006;91:48-53.

8. Essah PA, Nestler JE. The metabolic syndrome in polycystic ovary syndrome. J Endocrinol Invest 2006;29:270-80.

9. Carmina E, Lobo RA. Use of fasting blood to assess the prevalence of insulin resistance in women with polycystic ovary syndrome. Fertil Steril 2004;82:661-5.

10. Lin JF, Li X, Zhu MW. Exploration of the classification of polycystic ovarian syndrome. Zhonghua Fu Chan Ke Za Zhi 2006;41:684-8.

11. Chae SJ, Kim JJ, Choi YM, Hwang KR, Jee BC, Ku SY, et al. Clinical and biochemical characteristics of polycystic ovary syndrome in Korean women. Hum Reprod 2008;23:1924-31.

12. Kumar AN, Naidu JN, Satyanarayana U, Ramalingam K, Anitha M. Metabolic and endocrine characteristics of indian women with polycystic ovary syndrome. Int J Fertil Steril 2016;10:22-8.

13. Celik C, Abali R, Bastu E, Tasdemir N, Tasdemir UG, Gul A. Assessment of impaired glucose tolerance prevalence with hemoglobin $\mathrm{A}_{1} \mathrm{C}$ and oral glucose tolerance test in 252 Turkish women with polycystic ovary syndrome: a prospective, controlled study. Hum Reprod 2013;28:1062-8.

14. Zahiri Z, Sharami SH, Milani F, Mohammadi F, Kazemnejad E, Ebrahimi $\mathrm{H}$, et al. Metabolic syndrome in patients with polycystic ovary syndrome in Iran. Int J Fertil Steril 2016;9:490-6.

15. Ketel IJ, Stehouwer CD, Serne EH, Korsen TJ, Hompes PG, Smulders YM, et al. Obese but not normal-weight women with polycystic ovary syndrome are characterized by metabolic and microvascular insulin resistance. J Clin Endocrinol Metab 2008;93:3365-72.

16. Acien P, Quereda F, Matallin P, Villarroya E, Lopez-Fernandez JA, Acien $M$, et al. Insulin, androgens, and obesity in women with and without polycystic ovary syndrome: a heterogeneous group of disorders. Fertil Steril 1999;72:32-40.

17. Morciano A, Romani F, Sagnella F, Scarinci E, Palla C, Moro F, et al. Assessment of insulin resistance in lean women with polycystic ovary syndrome. Fertil Steril 2014;102:250-6.e3.

18. Arduc A, Saricam O, Dogan BA, Tuna MM, Tutuncu YA, Isik S, et al. Should insulin resistance be screened in lean hirsute women? Gynecol Endocrinol 2015;31:291-5.

19. Shah D, Rasool S. Polycystic ovary syndrome and metabolic syndrome: the worrisome twosome? Climacteric 2016;19:7-16.

20. Dunaif A. Insulin resistance and the polycystic ovary syndrome: mechanism and implications for pathogenesis. Endocr Rev 1997; 18:774-800.

21. Legro RS, Arslanian SA, Ehrmann DA, Hoeger KM, Murad MH, Pasquali $R$, et al. Diagnosis and treatment of polycystic ovary syndrome: an Endocrine Society clinical practice guideline. J Clin Endocrinol Metab 2013;98:4565-92.

22. Lee S, Choi S, Kim HJ, Chung YS, Lee KW, Lee HC, et al. Cutoff values of surrogate measures of insulin resistance for metabolic syndrome in Korean non-diabetic adults. J Korean Med Sci 2006; 21:695-700. 
23. Chun S. Relationship between early follicular serum estrone level and other hormonal or ultrasonographic parameters in women with polycystic ovary syndrome. Gynecol Endocrinol 2020;36: 143-7.

24. DeVane GW, Czekala NM, Judd HL, Yen SS. Circulating gonadotropins, estrogens, and androgens in polycystic ovarian disease. Am J Obstet Gynecol 1975;121:496-500.

25. Baird DT, Corker CS, Davidson DW, Hunter WM, Michie EA, Van Look PF. Pituitary-ovarian relationships in polycystic ovary syndrome. J Clin Endocrinol Metab 1977;45:798-801.

26. MacDonald PC, Rombaut RP, Siiteri PK. Plasma precursors of estrogen. I. Extent of conversion of plasma delta-4-androstenedione to estrone in normal males and nonpregnant normal, castrate and adrenalectomized females. J Clin Endocrinol Metab 1967;27: 1103-11.

27. de Kat AC, Broekmans FJ, Laven JS, van der Schouw YT. Anti-Müllerian Hormone as a marker of ovarian reserve in relation to cardio-metabolic health: a narrative review. Maturitas 2015;80:2517.

28. La Marca A, Orvieto R, Giulini S, Jasonni VM, Volpe A, De Leo V. Mullerian-inhibiting substance in women with polycystic ovary syndrome: relationship with hormonal and metabolic characteristics. Fertil Steril 2004;82:970-2.

29. Nardo LG, Yates AP, Roberts SA, Pemberton P, Laing I. The relationships between $\mathrm{AMH}$, androgens, insulin resistance and basal ovarian follicular status in non-obese subfertile women with and without polycystic ovary syndrome. Hum Reprod 2009;24:291723.

30. Chun S. 1-h Postprandial glucose level is related to the serum anti-Müllerian hormone level in women with polycystic ovary syn- drome. Gynecol Endocrinol 2015;31:815-8.

31. Rotterdam ESHRE/ASRM-Sponsored PCOS Consensus Workshop Group. Revised 2003 consensus on diagnostic criteria and longterm health risks related to polycystic ovary syndrome. Fertil Steril 2004;81:19-25.

32. Kim JJ, Chae SJ, Choi YM, Hwang SS, Hwang KR, Kim SM, et al. Assessment of hirsutism among Korean women: results of a randomly selected sample of women seeking pre-employment physical check-up. Hum Reprod 2011;26:214-20.

33. Kim JJ, Hwang KR, Oh SH, Chae SJ, Yoon SH, Choi YM. Prevalence of insulin resistance in Korean women with polycystic ovary syndrome according to various homeostasis model assessment for insulin resistance cutoff values. Fertil Steril 2019;112:959-66.e1.

34. Abdul-Ghani MA, Tripathy D, DeFronzo RA. Contributions of beta-cell dysfunction and insulin resistance to the pathogenesis of impaired glucose tolerance and impaired fasting glucose. Diabetes Care 2006;29:1130-9.

35. Nathan DM, Davidson MB, DeFronzo RA, Heine RJ, Henry RR, Pratley $\mathrm{R}$, et al. Impaired fasting glucose and impaired glucose tolerance: implications for care. Diabetes Care 2007;30:753-9.

36. Qiao Q, Jousilahti P, Eriksson J, Tuomilehto J. Predictive properties of impaired glucose tolerance for cardiovascular risk are not explained by the development of overt diabetes during follow-up. Diabetes Care 2003;20:2910-4.

37. Powers AC, Niswender KD, Evans-Molina C. Diabetes mellitus: diagnosis, classification, and pathophysiology [Internet]. New York: McGraw-Hill; 2018 [cited 2021 Oct 1]. Available from: http://accessmedicine.mhmedical.com/content.aspx?bookid = 2129\&sectionid $=192288322$. 